FEATURE

\title{
Relationship Between Nighttime Vital Sign Assessments and Acute Care Transfers in the Rehabilitation Inpatient
}

\author{
Anthony J. Pellicane ${ }^{1}$, MD \\ 1 Department of Physical Medicine and Rehabilitation at the Rehabilitation Institute of Michigan in the Detroit Medical Center, Wayne State \\ University, Detroit, MI, USA
}

\author{
Keywords \\ Vital signs; acute care transfer; patient \\ discharge; early diagnosis; rehabilitation; \\ rehabilitation nursing; sleep deprivation. \\ Correspondence \\ Anthony J. Pellicane, MD, Adventist \\ Rehabilitation Hospital of Maryland, 9909 \\ Medical Center Drive, Rockville, MD 20850. \\ E-mail: apellicane@hotmail.com \\ Accepted May 2, 2014 \\ doi: $10.1002 /$ rnj.169
}

\begin{abstract}
Purpose: To investigate the role of nighttime vital sign assessment in predicting acute care transfers (ACT) from inpatient rehabilitation.

Design: Retrospective chart review.

Methods: Fifty patients unexpectedly discharged to acute care underwent chart review to determine details of each ACT.

Findings: Seven of 50 ACT possessed new vital sign abnormalities at the 11 pm vital sign assessment the night before ACT. None of these seven underwent ACT during the night shift the abnormalities were detected. Two of 50 ACT were transferred between $11 \mathrm{pm}$ and 6:59 am; both demonstrating normal vital sign at the $11 \mathrm{pm}$ assessment. During study period, an estimated 5,607 $11 \mathrm{pm}$ vital sign assessments were performed.

Conclusions: Nighttime vital sign assessments do not seem to be a good screening tool for clinical instability in the rehabilitation hospital.

Clinical Relevance: Eliminating sleep disturbance is important to the rehabilitation inpatient as inadequate sleep hinders physical performance. Tailoring vital sign monitoring to fit patents' clinical presentation may benefit this population.
\end{abstract}

\section{Introduction}

The inpatient rehabilitation hospital is charged with delivering a variety of services to the individuals it cares for. Physicians, nurses, physical therapists, occupational therapists, speech language pathologists, dieticians, and psychologists are among those vying for the patient's time on a daily basis in this setting. Given the high number of daily patient-practitioner encounters, efficiency (achieved by streamlining necessary or eliminating unnecessary processes) is of paramount importance. Further, given that the goal of these encounters is to assist the patient to achieve the highest possible level of functional independence while eliminating any barriers that may limit functional progress, each encounter must have purpose and not interfere with the patient's advancement.

Sleep has important physiologic restorative functions and inadequate sleep can adversely affect neuroendocrine, cardiovascular, autonomic, and cognitive processes; behavior and mental health; (Zaharna \& Guilleminault, 2010) and physical performance (Souissi, Sesboue, Gauthier, Larue, \& Davenne, 2003). As the rehabilitation inpatient's day is filled with physical tasks geared toward improving function, sleep is of particular importance to this population. However, while positive functional outcomes are mandated in the rehabilitation setting, other factors are at play. These include safety and, the relative newcomer, patient satisfaction. As the Centers for Medicare and Medicaid Services has created an incentive fund for healthcare reimbursement based partly on patients' report of the hospital experience (Zamora, 2012) (with private payers likely to follow this model in the future (Zusman, 2012)), practitioners, hospitals, and hospital systems must adapt to improve patient satisfaction. Further, given that Southwell et al. reported that $50 \%$ of hospitalized patients were dissatisfied with settling and waking times and two-thirds of patients reported at least one sleep disturbance on the nights surveyed (Southwell 
\& Wistow, 1995), hospitals must focus on the elimination of iatrogenic sleep disturbances to maximize patient wellbeing. It should be noted that nurses attending to patients was the second most cited reason for sleep disturbances in the aforementioned study. When discussing the topic of sleep in the hospital setting, the interplay between patient safety, medical necessity, and patient satisfaction is interesting and complex.

This study investigates the need for nightly $11 \mathrm{pm}$ vital sign assessments in the acute inpatient rehabilitation setting by attempting to characterize these assessments' ability to predict acute care transfers (ACT) from the inpatient rehabilitation hospital. If it is determined that $11 \mathrm{pm}$ vital sign assessments are a poor screening tool for medical issues in the rehabilitation inpatient, perhaps elimination of these late checks should be considered to reduce sleep disturbances thereby improving physical performance, functional outcomes, and patient satisfaction.

\section{Methods}

This study was approved by the Wayne State University Institutional Review Board in Detroit, Michigan. ACT was defined as any unexpected discharge from the acute inpatient rehabilitation facility (Rehabilitation Institute of Michigan; Detroit, MI, USA) to any acute care facility. Hospital administration provided the patient name, financial identification number, and ACT date of the 50 most recent ACT at the time of request for this information. The 50 patients who were discharged unexpectedly underwent retrospective electronic medical record review to confirm the date of ACT and to determine the details surrounding the ACT (including etiology and documented subjective patient complaints related to the ACT), time of ACT, and the presence of new vital sign abnormalities at the $11 \mathrm{pm}$ per protocol vital sign assessment the day before the ACT.

Abnormal vital signs were defined as follows: systolic blood pressure $>200 \mathrm{mmHg}$ or change in systolic blood pressure $\pm 30 \mathrm{mmHg}$ compared to baseline, diastolic blood pressure $>100 \mathrm{mmHg}$ or change in diastolic blood pressure $\pm 15 \mathrm{mmHg}$ compared to baseline, heart rate $>100$ or $<60$ beats per minute (provided the patient was not tachycardic or bradycardic at baseline), respiratory rate $>24$ breaths per minute, temperature $>38.0{ }^{\circ} \mathrm{C}$ or $<35.7{ }^{\circ} \mathrm{C}$, and oxygen saturation $<92 \%$. The parameters used to define abnormal vital signs differed slightly from those previously used by Halm et al. (1998, 2003). Where applicable, baseline vital signs were established by taking the mean of all recorded values over the 2 days before the ACT. So, if a patient's mean heart rate was 55 over the 2 days before the ACT and the $11 \mathrm{pm}$ per protocol vital sign assessment the day before the ACT revealed bradycardia, this would not be considered an abnormal vital sign assessment. Instead, this was considered the patient's baseline.

Nursing shifts were defined as: day shift 7 am to $2: 59$ pm; evening shift $3 \mathrm{pm}$ to $10: 59 \mathrm{pm}$; night shift $11 \mathrm{pm}$ to 6:59 am; and were used to characterize time of ACT by nursing shift. ACT were also characterized as occurring between 8 am and 4:59 pm; 5 pm and 9:59 pm; or 10 pm and 7:59 am. These times were selected in an attempt to describe the ACT based on a nonshift worker's schedule and represented typical business hours, typical evening hours (before sleep), and typical night hours (after sleep), respectively.

\section{Results}

The 50 ACT studied occurred between August 30, 2012, and November 29, 2012. Forty-four patients accounted for the 50 ACT as six patients were discharged unexpectedly two times each from the acute inpatient rehabilitation facility. Reasons for ACT can be found in Table 1. In $88 \%(n=44)$ of the ACT, a new subjective patient complaint related to the reason for ACT was documented in the electronic medical record (for example, lower extremity pain due to osteomyelitis or nausea with vomiting due to gastric outlet obstruction). Thirty-four percent $(n=17)$ of ACT were associated with a sudden event (for example, unresponsiveness due to symptomatic bradycardia).

Eighty-two percent $(n=41)$ ACT occurred on a weekday, while $18 \%(n=9)$ occurred on a weekend. ACT per day of the week was as follows: Monday 10\% $(n=5)$, Tuesday $18 \%(n=9)$, Wednesday $18 \%(n=9)$, Thursday

Table 1 Reasons for Acute Care Transfer

\begin{tabular}{ll}
\hline \multicolumn{1}{c}{ Reason } & Percent $(n)$ \\
\hline Infectious disease & $40(20)$ \\
Cardiac & $18(9)$ \\
Venous thromboembolism & $12(6)$ \\
Gastrointestinal & $8(4)$ \\
Neurologic & $6(3)$ \\
Pulmonary & $6(3)$ \\
Renal & $4(2)$ \\
Oncologic & $4(2)$ \\
Undetermined & $2(1)$ \\
\hline
\end{tabular}


Table 2 Time of Acute Care Transfer

\begin{tabular}{lc}
\hline \multicolumn{1}{c}{ By Nursing Shift } & Percent $(n)$ \\
\hline Day (7 am-2:59 pm) & $58(29)$ \\
Evening (3 pm-10:59 pm) & $38(19)$ \\
Night (11 pm-6:59 am) & $4(2)$ \\
\hline By Nonshift Work Schedule & $74(37)$ \\
\hline Business hours (8 am-4:59 pm) & $22(11)$ \\
Evening hours (5 pm-9:59 pm) & $4(2)$ \\
Night hours (10 pm-7:59 am)
\end{tabular}

$14 \%(n=7)$, Friday $22 \%(n=11)$, Saturday $10 \%(n=5)$, and Sunday $8 \%(n=4)$. Specifics with regard to time of ACT can be found in Table 2. The two ACT occurring after $11 \mathrm{pm}$ warrant further description. In one, the $11 \mathrm{pm}$ per protocol vital sign assessment revealed no new abnormalities, however, was followed less than 1 hour later with sudden onset shortness of breath associated with tachypnea, oxygen desaturation, and worsening of baseline tachycardia $(101.5 \pm 6.0$ to $134.8 \pm 7.5$ beats per minute) Note, the $134.8 \pm 7.5$ beats per minute heart rate was calculated using four vital sign assessments subsequent to the shortness of breath complaint and were obtained at different time points over 1 hour. In the other nighttime ACT, the $11 \mathrm{pm}$ per protocol vital sign assessment revealed no abnormalities. Within 2 hours of this assessment, however, the patient developed sudden onset shortness of breath associated with oxygen desaturation. In the first nighttime ACT described, the patient was diagnosed with a pulmonary embolism; and, in the second, a mucous plug was deemed the etiology of the abnormalities.

Forty-eight of the 50 ACT (96\%) had an $11 \mathrm{pm}$ per protocol vital sign assessment recorded in the electronic medical record the day before unexpected discharge to acute care. Of these 48 ACT, 15\% $(n=7)$ demonstrated at least one new vital sign abnormality on the $11 \mathrm{pm}$ per protocol vital sign assessment. None of these seven patients, however, were discharged via ACT by the night shift staff who detected the abnormal vital sign(s). Three of the seven patients were discharged on the day shift (7 am to $2: 59 \mathrm{pm}$ ); and, four of the seven patients were discharged on the evening shift ( $3 \mathrm{pm}$ to $10: 59 \mathrm{pm}$ ) the day following the abnormal $11 \mathrm{pm}$ per protocol vital sign assessment. In addition, in only one of these seven patients did the abnormal vital sign(s) lead to a change in management. In that case, environmental modifications were made and blood work was ordered for the following morning in a spinal cord injured patient who developed a temperature $>38.0^{\circ} \mathrm{C}$. In the remaining six cases, there is no documentation that a physician altered the plan of care despite the newly detected and documented vital sign abnormalities.

During the aforementioned study period, the mean daily census for the entire hospital was $63.5 \pm 3.6$ patients. Using each day's actual daily census and assuming $96 \%$ of patients had an 11 pm per protocol vital sign assessment performed, an estimated 5,607 11 pm per protocol vital sign assessments were performed during the study period.

\section{Discussion}

Krumholtz (2013) has coined the term "post-hospital syndrome" which describes an acquired, transient period of vulnerability that is the direct result of a myriad of stressors (including but not limited to sleep deprivation) that the hospitalized patient endures. Understanding the deleterious effects of these stressors on patient outcomes and performance, the rehabilitation hospital should focus on their elimination. Specific to the rehabilitation inpatient, evidence suggests that sleep promotes off-line motor skill learning in healthy subjects as well as in individuals with brain damage (Siengsukon \& Boyd, 2009). Sleep is thought to provide a permissive environment that promotes various cellular and molecular mechanisms enabling the consolidation of memories. The stabilization or enhancement of a motor skill without additional physical practice results from memory consolidation occurring (for procedural memory) during REM sleep and stage 2 non-REM sleep and provides the foundation for the concept of off-line motor skill learning. Just as the literature supports that sleep benefits physical performance, it also supports that inadequate sleep adversely affects physical performance. For example, Reilly \& Piercy (1994) reported a significant decrease in submaximal weight-lifting tasks including biceps curl, bench press, leg press, and dead lift in partially sleep-deprived subjects that worsened with consecutive nights of sleep deprivation. Although some might attempt to argue that biceps curls and bench presses are less functionally relevant to the rehabilitation inpatient, it is clear that leg presses and dead lifts easily translate into important functional activities (specifically, transfers and ascending/descending stairs). Deficits in the performance of the muscle groups associated with these weight-lifting tasks due to inadequate sleep could lead to inefficient functional progress for the rehabilitation inpatient. In addition, as it has been reported that a variety of athletic activities are negatively impacted by sleep loss (Reilly \& Edwards, 2007) and as inpatient rehabilitation 
is a form of athletic training, sleep is of the upmost importance to this patient population. Thus, the rehabilitation hospital must not perpetuate the stressors that cause the posthospital syndrome and eliminate all unnecessary iatrogenic sleep disturbances whenever possible.

There is a paucity of literature investigating the relationship between clinical instability and adverse clinical events in the various postacute settings (Bernardini et al., 1995; Boockvar et al., 2003, 2004). Guerini et al. (2010), however, described a 6-fold higher risk of transfer to an acute care hospital from a rehabilitation ward when clinical instability (defined as one vital sign abnormality within 24 hours of rehabilitation admission) or delirium (determined using the Confusion Assessment Method) was present. When clinical instability and delirium were present concomitantly, this predicted a 10 -fold higher risk for transfer to an acute care hospital. As the mean length of stay for patients with clinical instability and/or delirium in the study was greater than 26 days on the rehabilitation ward, this suggests that, while vital sign abnormalities predict transfer to acute care, they do not necessarily identify those patients with an immediate need for transfer. Demonstratively, in the study described here, of the seven patients with at least one new vital sign abnormality on 11 pm per protocol vital sign assessment, none were discharged via ACT by the night shift staff who detected the abnormal vital sign(s). Relatedly, in the two nighttime ACT described earlier, the $11 \mathrm{pm}$ per protocol vital sign assessments were without new abnormalities but were quickly and unexpectedly followed by sudden onset clinical change and new vital sign abnormalities. Thus, it seems that abnormal vital signs do not always predict an immediate need for $\mathrm{ACT}$, and normal vital signs do not necessarily ensure clinical stability. As a result, a call to act on abnormal vital signs when first noted to prevent the "delayed" ACT is in order. Further, due to the unpredictability of the rehabilitation inpatient, focusing and acting on vital sign trends may be more advantageous than arbitrarily assessing vital signs at times that may inhibit a patient's ability to get the appropriate amount and quality of sleep.

The scientific community lacks strong evidence with regard to sleep promotion and sleep disturbance prevention in the hospital setting (Hellstrom \& Willman, 2011). Common sense would suggest that eliminating the disturbance of a sleeping inpatient with an unnecessary nighttime vital sign assessment would improve sleep outcomes. For example, in an acute care hospital, LaReau et al. (2008) implemented a sleep protocol that, pertinent to the study described here, clustered nursing activities and delayed or eliminated unnecessary interruptions (among other nonpharmacologic sleep interventions). The experimental group enjoyed significantly better sleep quality and ability to remain asleep and used fewer sleep medications compared to the control group with these nonpharmacologic sleep interventions. In the study described here, as an estimated 5,607 nighttime per protocol vital sign assessments were performed during the study period and as only seven of the ACT in this study possessed abnormal nighttime vital signs, one must question the dogmatic prescription of nighttime vital sign assessments in the rehabilitation inpatient (particularly if these assessments are considered screening tools for underlying disease (Abram \& Valesky; Andermann, Blancquaert, Beauchamp, \& Dery, 2008; Wilson \& Jungner, 1968)). Further, as track and trigger systems outside of critical care settings have not been determined to reliably identify at-risk patients (Gao et al., 2007; Jansen \& Cuthbertson, 2010), reconsidering rigid vital sign assessment protocols in the rehabilitation inpatient is not unreasonable. Of course, this is not to suggest that vital signs are unimportant in the rehabilitation hospital. Rather, this is to suggest that vital signs should be tailored to a patient's needs based on clinical judgment (Gao et al., 2007; Kyriacos, Jelsma, \& Jordan, 2011; Odell, Victor, \& Oliver, 2009) particularly given that $88 \%$ of ACT in the study described here were associated with subjective patient complaints. Those patients with a constellation of vital sign abnormalities and other worrisome clinical signs and symptoms may warrant more frequent monitoring, whereas those without a concerning assortment of subjective and objective findings might benefit from less frequent (or less disruptive) monitoring. For example, as tachypnea is a particularly important indicator of an at-risk patient and is the most common abnormality found in critical illness (Goldhill, McNarry, Hadjianastassiou, \& Tekkis, 2004; Goldhill, Worthington, Mulcahy, Tarling, \& Sumner, 1999; Jevon \& Ewens, 2012), perhaps respiratory rate should be the sole measured nighttime vital sign in rehabilitation inpatients deemed clinically stable as respiratory rate can be measured without disturbing a patient's sleep.

\section{Limitations}

Due to the retrospective nature of this study, in depth analysis of each ACT, for example by way of discussion of the events surrounding each ACT with involved parties, was not possible. Thorough review of the electronic medical record, however, at least mitigated this shortcoming. 


\section{Key Practice Points}

- An infectious disease issue accounted for $40 \%$ of the acute care transfers from the rehabilitation hospital.

- Representing the unpredictability of the rehabilitation inpatient, in two of the 50 ACT studied, normal nighttime vital sign assessments were soon followed by sudden onset subjective and objective decline.

- Per protocol nighttime vital sign assessments do not seem to be a good screening tool for clinical instability in the rehabilitation inpatient.

- As inadequate sleep hinders physical performance, eliminating iatrogenic sleep disturbances such as nighttime vital sign assessments may benefit the rehabilitation inpatient.

Relatedly, based on documentation, it appears that in only one of seven instances of abnormal 11 pm per protocol vital sign assessments was a physician contacted. Of course, the possibility of an undocumented conversation is possible. The retrospective nature of this study prevents knowledge of such encounters.

\section{Conclusions}

Sleep is of paramount importance to the rehabilitation inpatient as adequate sleep is necessary for optimal physical performance. As such, any iatrogenic cause of sleep disturbance in the rehabilitation hospital should be eliminated or, at the very least, minimized. Of course, patient safety through appropriate monitoring is also of great importance in the inpatient rehabilitation setting. On the basis of the information described in this study coupled with other studies that suggest against track and trigger systems outside of the critical care setting, we must challenge the notion that all patients require the same number of daily vital sign assessments in the acute inpatient rehabilitation hospital. It is understood that the monitoring of vital signs in the rehabilitation hospital is necessary. It can be suggested that those inpatients deemed more clinically stable may benefit from less frequent (or less disruptive) assessments to optimize patient safety, functional outcomes, and patient satisfaction.

\section{References}

Abram, E., \& Valesky, W. Screening and diagnostic tests. Retrieved October 20, 2013, from http://emedicine. medscape.com/article/773832-overview
Andermann, A., Blancquaert, I., Beauchamp, S., \& Dery, V. (2008). Revisiting Wilson and Jungner in the genomic age: A review of screening criteria over the past 40 years. Bulletin of the World Health Organization, 86(4), 317-319.

Bernardini, B., Meinecke, C., Pagani, M., Grillo, A., Fabbrini, S., Zaccarini, C. et al. (1995). Comorbidity and adverse clinical events in the rehabilitation of older adults after hip fracture. Journal of the American Geriatrics Society, 43(8), 894-898.

Boockvar, K.S., Halm, E.A., Litke, A., Silberzweig, S.B., McLaughlin, M., Penrod, J.D. et al. (2003). Hospital readmissions after hospital discharge for hip fracture: Surgical and nonsurgical causes and effect on outcomes. Journal of the American Geriatrics Society, 51(3), 399-403.

Boockvar, K.S., Litke, A., Penrod, J.D., Halm, E.A., Morrison, R.S., Silberzweig, S.B. et al. (2004). Patient relocation in the 6 months after hip fracture: Risk factors for fragmented care. Journal of the American Geriatrics Society, 52(11), 1826-1831.

Gao, H., McDonnell, A., Harrison, D.A., Moore, T., Adam, S., Daly, K. et al. (2007). Systematic review and evaluation of physiological track and trigger warning systems for identifying at-risk patients on the ward. Intensive Care Medicine, 33(4), 667-679.

Goldhill, D.R., McNarry, A.F., Hadjianastassiou, V.G., \& Tekkis, P.P. (2004). The longer patients are in hospital before intensive care admission the higher their mortality. Intensive Care Medicine, 30(10), 1908-1913.

Goldhill, D.R., Worthington, L., Mulcahy, A., Tarling, M., \& Sumner, A. (1999). The patient-at-risk team: Identifying and managing seriously ill ward patients. Anaesthesia, 54(9), 853-860.

Guerini, F., Frisoni, G.B., Morghen, S., Speciale, S., Bellelli, G., \& Trabucchi, M. (2010). Clinical instability as a predictor of negative outcomes among elderly patients admitted to a rehabilitation ward. Journal of the American Medical Directors Association, 11(6), 443-448.

Halm, E.A., Fine, M.J., Marrie, T.J., Coley, C.M., Kapoor, W.N., Obrosky, D.S. et al. (1998). Time to clinical stability in patients hospitalized with community-acquired pneumonia: Implications for practice guidelines. JAMA, 279 (18), 1452-1457.

Halm, E.A., Magaziner, J., Hannan, E.L., Wang, J.J., Silberzweig, S.B., Boockvar, K. et al. (2003). Frequency and impact of active clinical issues and new impairments on hospital discharge in patients with hip fracture. Archives of Internal Medicine, 163(1), 108-113.

Hellstrom, A., \& Willman, A. (2011). Promoting sleep by nursing interventions in health care settings: A systematic review. Worldviews on Evidence-Based Nursing, 8(3), 128142. 
Jansen, J.O., \& Cuthbertson, B.H. (2010). Detecting critical illness outside the ICU: The role of track and trigger systems. Current Opinion in Critical Care, 16(3), 184190.

Jevon, P., \& Ewens, B. (2012). Monitoring the critically ill patient. (3rd ed.). Chinchester, West Sussex, UK: Blackwell Publishing Ltd.

Krumholz, H.M. (2013). Post-hospital syndrome-an acquired, transient condition of generalized risk. New England Journal of Medicine, 368(2), 100-102.

Kyriacos, U., Jelsma, J., \& Jordan, S. (2011). Monitoring vital signs using early warning scoring systems: A review of the literature. Journal of Nursing Management, 19(3), 311-330.

Lareau, R., Benson, L., Watcharotone, K., \& Manguba, G. (2008). Examining the feasibility of implementing specific nursing interventions to promote sleep in hospitalized elderly patients. Geriatric Nursing, 29(3), 197-206.

Odell, M., Victor, C., \& Oliver, D. (2009). Nurses' role in detecting deterioration in ward patients: Systematic literature review. Journal of Advanced Nursing, 65(10), 19922006.

Reilly, T., \& Edwards, B. (2007). Altered sleep-wake cycles and physical performance in athletes. Physiology and Behavior, 90 (2-3), 274-284.
Reilly, T., \& Piercy, M. (1994). The effect of partial sleep deprivation on weight-lifting performance. Ergonomics, 37 (1), 107-115.

Siengsukon, C.F., \& Boyd, L.A. (2009). Does sleep promote motor learning? Implications for physical rehabilitation Physical Therapy, 89(4), 370-383.

Souissi, N., Sesboue, B., Gauthier, A., Larue, J., \& Davenne, D. (2003). Effects of one night's sleep deprivation on anaerobic performance the following day. European Journal of Applied Physiology and Occupational Physiology, 89(3-4), 359-366.

Southwell, M.T., \& Wistow, G. (1995). Sleep in hospitals at night: Are patients' needs being met? Journal of Advanced Nursing, 21(6), 1101-1109.

Wilson, J., \& Jungner, G. (1968). Principles and practice of screening for disease. The Journal of the Royal College of General Practitioners, 16(4), 318.

Zaharna, M., \& Guilleminault, C. (2010). Sleep, noise and health: Review. Noise Health, 12(47), 64-69.

Zamora, D. (2012). Using patient satisfaction as a basis for reimbursement: Political, financial, and philosophical implications. Creative Nursing, 18(3), 118-123.

Zusman, E.E. (2012). HCAHPS replaces Press Ganey survey as quality measure for patient hospital experience.

Neurosurgery, 71(2), N21-N24. 